\title{
Equation of State of Crude Oil Samples
}

\author{
Abraham K. George*, Ram N Singh and Sayyadul Arafin
}

Physics Department, Sultan Qaboos University, P.O. Box 36, Al Khodh 123, Muscat, Oman

\begin{abstract}
Technological advancement in exploration, refinement and enhanced recovery of hydrocarbon fluids heavily depend upon the thermo-physical properties of these fluids under different temperature and pressure conditions. A direct measurement at in-situ conditions is quite difficult; therefore we have utilized thermodynamic equations to extrapolate these thermo-physical properties at ambient conditions. The basic ingredients are density and ultrasound velocity which we have measured in the laboratory as a function of temperature for light and heavy crude oils procured from different wells of Oman. The densities of the samples were measured as a function of temperature using a precision density meter and the sound velocity using an ultrasound interferometer. To understand the variation of physical properties on mixing we have studied a 50:50 mixture of light and heavy oils. These data, in turn, were used to develop the equation of state. Our computed equation of state conforms reasonably well to the in-situ reservoir conditions.
\end{abstract}

Keywords: Crude oil; API number; Ultrasound velocity; Equation of state

\section{Introduction}

Crude oil is a complex mixture of many different chemical components, mainly organic compounds (hydrocarbons) which usually make up about $95 \%$ of it. The remaining compounds of crude oil are made up of small amounts of oxygen, nitrogen, sulfur and small amount of metals such as iron, vanadium, nickel and chromium. The composition of crude oil depends on the origin and conditions of formation. Physical properties and chemical composition vary $[1,2]$ from one reservoir to another and even between different depths in the same well.

The identification [3-7] of the physical properties of a particular crude oil is essential to provide a basis for exploration, transportation and refinement process. These are widely used in crude oil processing such as refining operations, optimization of equipment design and in-situ reservoir simulation. Producers and refiners depend on the basic physical parameters like density, ultrasonic velocity, acoustic impedance, elastic modulii and the equation of state. The resulting equation of state characterizes the fluid correctly and reproduces properties such as saturation pressure and the liquid phase density. Such information is useful in enhanced recovery of crude oil from deep well.

In our work, we have used two different experimental techniques:

- Ultrasound interferometer to determine the velocity of propagation of compression waves over a wide range of temperature ranging from $20^{\circ} \mathrm{C}$ to $70^{\circ} \mathrm{C}$.

- An Anton Paar density meter to measure the density at the corresponding temperatures.

These functions are later used in thermodynamics equations to determine the thermo-physical properties and its variation as a function of temperature. It is also our interest to measure the properties of the mixture of crude oils having different API gravity. This helps us to understand the variation in the physical properties of crude oils on mixing. Particularly, we have studied such variation on mixing the light and heavy crude oils.

\section{Experimental}

\section{Density of crude oil samples}

The density of the samples was measured using an Anton Paar density meter (DMA 5000). The unit consists of a U-shaped oscillating tube and a system for electronic excitation, frequency counting and display. The injected sample volume was kept constant and vibrated. The density was calculated based on a measurement of the sample oscillation period and temperature. The temperature was controlled to $\pm 0.01^{\circ} \mathrm{C}$ during the measurement using a built in thermostat. By measuring the damping of the U-tube's oscillations caused by the viscosity of the filled-in sample, the instrument automatically corrects the viscosity related errors. The above instrument enables density to be measured to an accuracy of $\pm 5 \times 10^{-3} \mathrm{~kg} \mathrm{~m}^{-3}$.

The petroleum crude oil samples utilized in this work were procured from two different fields, both located in the north of Sultanate of Oman. In order to investigate the change in properties due to mixing, we prepared a 50:50 by weight mixture of the two oils. The oils mix readily and form a homogeneous mixture. The measured densities as a function of temperature are presented in Table 1.

The density of the mixture is found to be almost the average value of the density of the light and heavy oils. This is because the density depends on the mass and the mass is an intrinsic physical quantity and since we made 50:50 by weight mixture, we expect this result. However, there are larger deviations at higher temperatures which may be due to vaporization some components of the oil samples.

*Corresponding author: Abraham K George, Physics Department, Sultan Qaboos University, P.O. Box 36, Al Khodh 123, Muscat, Oman, E-mail: akgeorge@squ.edu.om

Received October 16, 2013; Accepted November 28, 2013; Published December 06, 2013

Citation: George AK, Singh RN, Arafin S (2013) Equation of State of Crude Oil Samples. J Pet Environ Biotechnol 4: 162. doi:10.4172/2157-7463.1000162

Copyright: (c) 2013 George AK, et al. This is an open-access article distributed under the terms of the Creative Commons Attribution License, which permits unrestricted use, distribution, and reproduction in any medium, provided the original author and source are credited. 


\begin{tabular}{|c|c|c|c|}
\hline Temperature $\left({ }^{\circ} \mathrm{C}\right)$ & \multicolumn{3}{|c|}{ Density $\left( \pm 0.005 \mathrm{~kg} / \mathrm{m}^{3}\right)$} \\
\hline & Light oil & Heavy oil & Medium oil $(50: 50$ mixture) \\
\hline 20 & 832.991 & 952.687 & 890.499 \\
\hline 25 & 829.500 & 949.462 & 887.262 \\
\hline 30 & 826.006 & 946.199 & 883.950 \\
\hline 35 & 822.494 & 942.882 & 880.581 \\
\hline 40 & 818.954 & 939.441 & 877.200 \\
\hline 55 & 815.482 & 936.214 & 873.773 \\
\hline 55 & 811.979 & 933.050 & 870.422 \\
\hline 60 & 808.477 & 929.882 & 866.952 \\
\hline 65 & 804.977 & 926.721 & 862.631 \\
\hline 70 & 801.471 & 923.562 & 857.632 \\
\hline & 797.960 & 920.408 & 851.150 \\
\hline
\end{tabular}

Table 1: Data of the density in light, heavy and the mixture of oil samples at different temperatures.

\section{API gravity}

Crude oil samples can be classified as light, medium or heavy according to their API gravity. API is a specific gravity scale developed by the American Petroleum Institute for measuring the relative density of various petroleum liquids [8]. It is defined as:

$$
A P I=\frac{141.5}{\rho}-131.5
$$

where $\rho$ is the density of crude oil in $\left(\mathrm{g} / \mathrm{cm}^{3}\right)$ at temperature $\mathrm{T}=$ $15.6^{\circ} \mathrm{C}$ and at atmospheric pressure. We have used our density data to measure the API for our samples. The API gravity of our samples are:

Sample-1: API = 35.9

Sample-2: API = 15.9

Crude oil is classified [9] as light if the API is greater than 31.1 and as heavy if the API is less than 22.3. Between 22.3 and 31.1, the crude oil is classified as medium. Following this classification for our samples, Sample- 1 is classified as light crude oil whereas sample- 2 is classified as heavy. We also used our measured density to determine the API gravity of the crude oil mixture:

Mixture (of light and heavy crude oil): API $=21.5$

As per specifications discussed earlier, the mixture falls under the category of medium oil. It can be seen that the measured API of the mixture deviate considerably from the average value of the mixture, i.e.,

Excess API of the mixture $=$ Measured API of the mixture $-[0.5$ API of Sample $1+0.5$

$$
\text { API of sample 2] }=-4.4(\mathrm{API}) \text {. }
$$

API of the mixture sample decreases on mixing and tends towards heavier side. Hereafter we call the three oils as light (sample 1), medium (mixture) and heavy (sample 2).

\section{Ultrasound velocity}

Velocity of compressional waves $v(T)$, was measured as a function of temperature using an ultrasonic interferometer, which consists of: (i) a high frequency generator, and (ii) a measuring cell. The high frequency generator is designed to excite the quartz crystal at the bottom of the measuring cell at its resonant frequency to generate ultrasonic waves in the experimental liquid. The frequency can be varied from 1 to $5 \mathrm{MHz}$; in our experiment, the ultrasound velocity was measured at a frequency of $1 \mathrm{MHz}$. The measuring cell is connected to the output terminal of the high frequency generator through a shielded cable. It is a specially designed double walled jacket which enables the temperature of the experimental liquid maintained constant by circulating water at a desired temperature. The temperature of the cell was controlled to $\pm 0.1^{\circ} \mathrm{C}$ using a circulation thermostat (Haake D8). The measuring cell was kept in an insulated jacket to minimize the heat loss during he measurement. The quartz crystal is fixed at the bottom of the cell. At the top, a fine micrometer is attached to a movable metallic plate reflector which is kept parallel to the quartz crystal. The micrometer can be used to raise or lower the reflector plate in the experimental liquid through known distances.

The principle used in the measurement of velocity is based on the accurate determination of the wavelength in the medium. The waves generated by the quartz crystal are reflected by the metallic plate reflector kept parallel to it. The reflector in the measuring cell is slowly moved either up or down. If the separation between the crystal and the reflector is exactly a whole multiple of the sound wavelength, standing waves are formed in the medium. This acoustic resonance gives rise to an electrical reaction on the generator driving the quartz crystal and the anode current of the generator becomes a maximum. If the distance is increased or decreased and the variation is exactly half wavelength or multiple of it, the anode current becomes a maximum. The reflector position is varied for ' $n$ ' maxima and its position is noted again. From the known distance $(d)$ moved by the reflector, the wavelength of the ultrasonic wave is estimated using the relation, $d=n(\lambda / 2)$. To minimize the errors, this process is repeated for several peaks and the average value of $\lambda$ is obtained and hence the velocity, $v=f \lambda$, is determined. The measured data of temperature dependence of ultrasound velocity is presented in Table 2 .

In general, the reason for decrease in velocity with temperature may be due to the fact that inter-atomic distance increases with temperature because of thermal expansion which in turn decreases the velocity. This is further supported by an idea that sound wave travels faster in the close-packed arrangement of atoms than in the loosepacked arrangement of atoms. Our data of ultrasonic velocity in crude oils clearly indicated that the motion of sound wave largely depends on internal molecular dynamics in each oil. Since the velocity of sound is larger in heavy crude oil, which may be an indication for the existence of stronger intermolecular interaction between a certain molecules in heavy crude oil. This also explains why the velocity of sound in mixture crude oil is not the average between the corresponding values in light and heavy crude oils.

\begin{tabular}{|c|c|c|c|}
\hline & \multicolumn{3}{|c|}{ Ultrasound velocity $( \pm 1 \mathrm{~m} / \mathrm{s})$} \\
\hline & Light oil & Heavy oil & $\begin{array}{c}\text { Medium oil }(50: 50 \\
\text { mixture })\end{array}$ \\
\hline 20 & 1420 & 1496 & 1361 \\
\hline 25 & 1403 & 1480 & 1342 \\
\hline 30 & 1385 & 1465 & 1323 \\
\hline 35 & 1368 & 1449 & 1308 \\
\hline 40 & 1349 & 1432 & 1292 \\
\hline 45 & 1334 & 1419 & 1278 \\
\hline 50 & 1316 & 1404 & 1260 \\
\hline 55 & 1301 & 1387 & 1243 \\
\hline 60 & 1288 & 1371 & 1223 \\
\hline 65 & 1267 & 1354 & 1210 \\
\hline 70 & 1257 & 1337 & 1193 \\
\hline
\end{tabular}

Table 2: Data of velocity of sound in light, heavy and the mixture of oil samples at different temperatures. 
Citation: George AK, Singh RN, Arafin S (2013) Equation of State of Crude Oil Samples. J Pet Environ Biotechnol 4: 162. doi:10.4172/21577463.1000162

\section{Results and Discussion}

The experimentally measured quantities of the ultrasound velocity and density form the basis for the thermo-physical characterization of crude oil through thermodynamic relations. This facilitated the evaluation of compressibility and volume expansion and the Equation of State (EOS) of crude oil has been set up using these data. It is a constitutive equation describing the state of matter under a given set of physical conditions. EOS provides a mathematical relationship between two or more state functions associated with the matter, such as its temperature, pressure, volume, or internal energy. EOS is useful in describing the properties of fluids, mixtures of fluids, solids, and even the interior of stars.

The velocity of compressional waves and density of liquid hydrocarbon measured as a function of temperature offer a potentially favorable route for the in-situ characterization of reservoir liquid in a given well. If the equation of state is solved for $P$ which is a function of temperature $T$ and volume $\Omega, P=$ function of $(T, \Omega)$, then

$$
d P=\left(\frac{\partial P}{\partial T}\right)_{\Omega} d T+\left(\frac{\partial P}{\partial \Omega}\right)_{T} d \Omega .
$$

At constant volume,

$$
d P=\left(\frac{\partial P}{\partial T}\right)_{\Omega} d T
$$

From the basic definition of the coefficient of volume, $\beta=\frac{1}{\Omega}\left(\frac{d \Omega}{d T}\right)_{P}=-\frac{1}{\rho}\left(\frac{\partial \rho}{\partial T}\right)_{P}$ and the isothermal compressibility, $\kappa_{T}\left[=\frac{1}{\Omega}\left(\frac{d \Omega}{d P}\right)_{T}\right]$, one readily has,

$$
d P=\frac{\beta}{\kappa_{t}} d t
$$

$\beta$ has been determined from the measured data of density as a function of temperature. $\kappa_{T}$ is determined from $\kappa_{S}$, the adiabatic compressibility and the ratio $\left(\gamma=C_{P} / C_{V}\right)$ of specific heats,

$$
\frac{\kappa_{t}}{\kappa_{S}}=\gamma ; \quad \kappa_{\mathrm{S}}=v^{-2} \rho^{-1}
$$

Sound velocity $v$ is taken from Table 2 . The values of $\gamma$ were determined [10] from the measured values of the specific heat. It was found that $\gamma$ is almost independent of temperature. $\gamma$ is found to be 1.22 at $20^{\circ} \mathrm{C}$ which decreases to 1.18 at $70^{\circ} \mathrm{C}$. It was noted that a decrease of roughly $3 \%$ is within the limits of the experimental errors. We have used the average value of $\gamma$ for light oil as 1.21, for heavy oil as 1.13 and for the mixture as 1.20

Integrating the equation (4) form the reference state $\left(P_{r}, t_{r}\right)$ to any other state $(P, t)$ one gets,

$$
P=P_{r}+\int_{t_{r}}^{t} \frac{\beta}{\kappa_{t}} d t
$$

The right hand side of the integral can be evaluated from our experimental data of $\beta$ and $\kappa_{t}$. For small range of temperature $(\Delta t)$, only little error is introduced assuming that both $\beta$ and $\kappa_{t}$ are constants. Hence,

$$
(\Delta P / \Delta t)
$$

$(\Delta P / \Delta t)$ Computed from equation (7) from the known values of $\beta$ and $\kappa_{t}$ at a given $t$ are plotted as a function of temperature in Figure

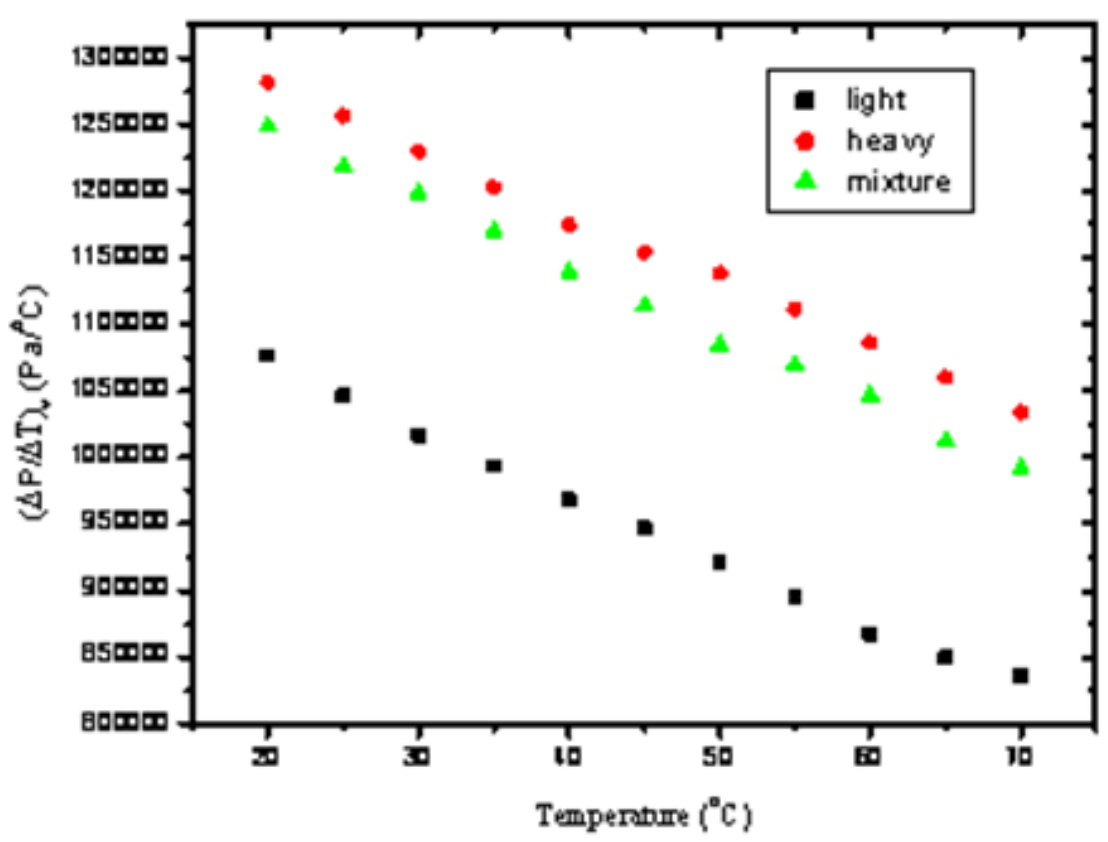

Figure 1: Variation of $(\Delta P / \Delta t)_{\mathrm{V}}$ with temperature for light, heavy and mixture of crude oils. 
Citation: George AK, Singh RN, Arafin S (2013) Equation of State of Crude Oil Samples. J Pet Environ Biotechnol 4: 162. doi:10.4172/21577463.1000162

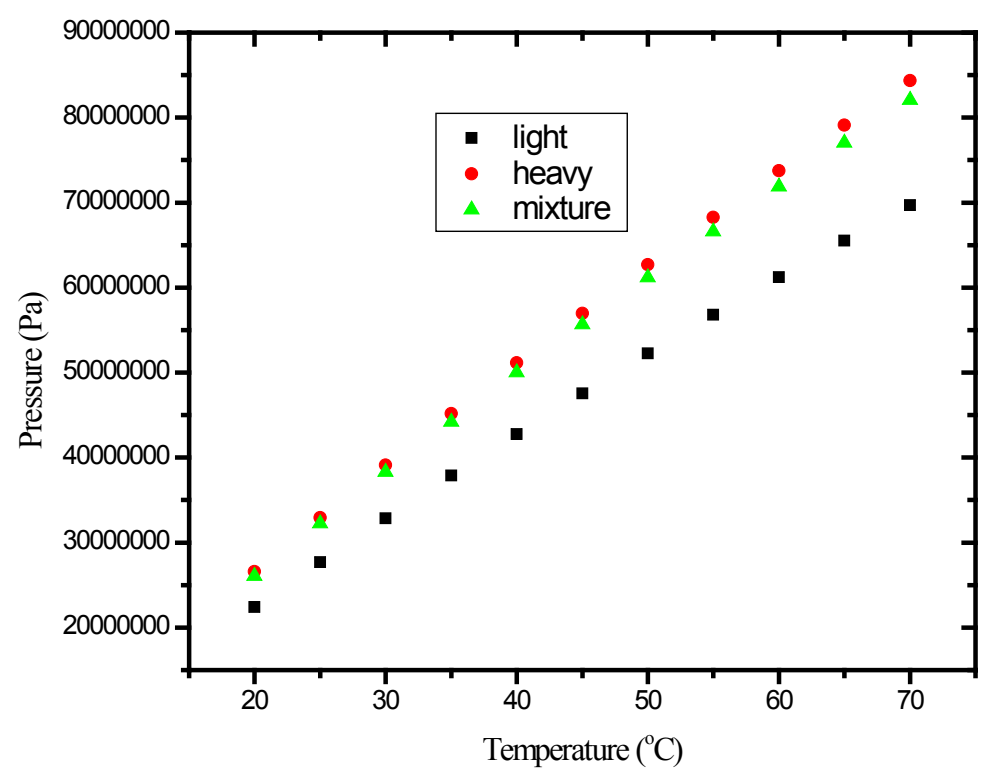

Figure 2: Computed pressure against temperature for light, heavy and mixture of crude oil.

1. The values of $(\Delta P / \Delta t)$ can readily fitted into equations:

$$
\begin{aligned}
& \text { Light oil }(\Delta P / \Delta t)=1.1638 \times 10^{6}-4845.593 \mathrm{t} \\
& \text { Heavy oil }(\Delta P / \Delta t)=1.3734 \times 10^{6}-4849.436 \mathrm{t} \\
& \text { Mixture }(\Delta P / \Delta t)=1.3495 \times 10^{6}-5112.224 \mathrm{t}
\end{aligned}
$$

With $(\Delta P / \Delta t)$ as in equations (8) to (10), the integral equation (6) is evaluated between the reference state $\left(P_{r}, t_{r}\right)$ to any other state $(P$, $t$ ) which yields expressions for the pressure,

$$
\begin{aligned}
& \text { Light oül } P=P r+1.1638 \times 10^{6}\left(\mathrm{t}_{\mathrm{r}}\right)-2422.797\left(\mathrm{t}^{2}-\mathrm{t}_{\mathrm{r}}^{2}\right) \\
& \text { Heavy oül } P=P \mathrm{r}+1.3734 \times 10^{6}\left(\mathrm{t}_{\mathrm{r}}\right)-2424.718\left(\mathrm{t}^{2}-\mathrm{t}_{\mathrm{r}}^{2}\right) \\
& \text { Mixture } P=P \mathrm{r}+1.3495 \times 10^{6}\left(\mathrm{t}_{\mathrm{r}}\right)-2556.112\left(\mathrm{t}^{2}-\mathrm{t}_{\mathrm{r}}^{2}\right)
\end{aligned}
$$

The $P-T$ variations are shown in Figure 2. The pressure is found to increase with increasing temperature. The $\mathrm{P}$-T variation of heavy oil and the mixture are closer to each other, particularly in the low temperature close to room temperature. At higher temperature the pressure of the mixture becomes less than heavy oil. Heavy oil fluids contain larger concentration of high molecular weight components and hence exhibit large pressure at any given temperature. At any given temperature the pressure of light oil is smaller than the heavy oil. The difference increases with increasing temperature. It is worth mentioning that our suggested equations (11-13) yields results very close to those obtained directly from similar oil fields. One of the known values [10] of the temperature and pressure supplied by Petroleum Development Oman (PDO) are $89^{\circ} \mathrm{C}$ and $89.5 \mathrm{MPa}$. We have used equations $(11-13)$ to calculate the reservoir pressure corresponding to its temperature at $89^{\circ} \mathrm{C}$. Our computed values are: $84.6 \mathrm{MPa}$ (light oil), $102.5 \mathrm{MPa}$ (heavy oil) and $100.8 \mathrm{MPa}$ (mixture). The value supplied by PDO is quite close to our value of the light oil.

\section{Summary and Conclusions}

The density and ultrasound velocity of light, medium and heavy oil samples were measured as a function of temperature. These are found to decrease linearly with temperature. These data were utilized to evaluate the thermo-physical properties utilizing thermodynamic relations. The data made it possible to set up the EOS, which is in agreement with in situ crude oil reservoir conditions. Our present approach of using the basic laboratory data for developing the EOS of hydrocarbon fluids is very useful in reservoir engineering. It can be utilized for calculation of the thermodynamic data and phase behavior including the volumetric analysis, the vapor/liquid equilibrium and thermal properties of crude oils.

\section{References}

1. Petroleum Manufacturing Orientation Course (1997) Mobil Research and Engineering. Paulsboro, New Jersey USA.

2. McCain WD (1990) The Properties of Petroleum Fluids. PennWell Books, USA

3. Singh RN, George AK, Arafin S (2006) Specific heat ratio, Grüneisen parameter and Debye temperature of crude oil. J Phys D Appl Phys 39: 1220-1225.

4. Riazi MR, Al-Adwani HA, Bishara A (2004) The impact of characterization methods on properties of reservoir fluids and crude oils: options and restrictions. J Petrol Sci Eng 42: 195-207.

5. Ball SJ, Goodwin ARH, Trusler JPM (2002) Phase behavior and physical properties of petroleum reservoir fluids from acoustic measurements. J Petrol Sci Eng 34: 1-11.

6. Wang Z, Nur AM, Batzle ML (1990) Acoustic Velocities in Petroleum Oils. J Petrol Technol 42: 192-200.

7. Zhijing W, Amos N (1991) Ultrasonic velocities in pure hydrocarbons and mixtures. J Acoust Soc Am 89: 2725-2730.

8. Kayan CF (2012) Systems Of Units: National And International Aspects. Literary Licensing, USA.

9. Amyx JW, Bass DM, Whiting RL (1960) Petroleum Reservoir Engineering: Physical properties. McGraw-Hill, USA.

10. Heward A and Al-Mahrooqi S (2005) Petroleum Development Oman, Personal Communication. 